\title{
Hybrid Heuristic Technique for Optimal Distributed Generation Integration in Distribution Systems
}

\author{
Mohamed Darfoun ${ }^{1}$, Huda Hosson ${ }^{2}$ \\ ${ }^{1}$ Electrical Engineering Department, College of Engineering Technology, Houn, Libya \\ ${ }^{2}$ Electric and Computer Engineering, University of Nebraska, Lincoln, NE, USA
}

Email address:

m.darfoun@ceh.edu.ly (M. Darfoun), hhosson2@huskers.unl.edu (H. Hosson)

\section{To cite this article:}

Mohamed Darfoun, Huda Hosson. Hybrid Heuristic Technique for Optimal Distributed Generation Integration in Distribution Systems. International Journal of Data Science and Analysis. Vol. 7, No. 3, 2021, pp. 82-88. doi: 10.11648/j.ijdsa.20210703.15

Received: May 8, 2021; Accepted: June 7, 2021; Published: June 16, 2021

\begin{abstract}
Integration of Distributed Generations (DGs) in distribution systems receives great attention nowadays due to its numerous benefits, the most important of which are reducing the overall power losses and improving voltage profile in distribution systems. In order to enhance the performance of the network, the DG units must be installed at optimal placement and sizing. Solution techniques for DG placement rely on various optimization methods. In this paper, a hybrid heuristic technique is proposed to solve the optimization problem for a single DG unit using two heuristic tests performed in two stages. In the first stage, a sensitivity test is used to determine the candidate location for DG placement. Then in the second stage, the optimal size is identified using a curve fitting test. A comprehensive analysis is performed in order to validate the results of the proposed technique. Both techniques have been tested on IEEE 33-bus and 69-bus radial distribution test systems. The obtained results show that although the comprehensive analysis can achieve slightly greater power loss reduction and voltage profile improvement, it requires a large number of tests that is proportional to the size of the distribution system. On the other hand, the proposed technique can achieve comparable results using a small fixed number of tests for any system, which means that this technique reduces the solution search space i.e., the computational demand and convergence time, while maintaining satisfactory results.
\end{abstract}

Keywords: Distributed Generation, Optimization, Radial Network, Fitting Curve

\section{Introduction}

It is known that the electrical energy that is fed to the electric power networks does not completely reach the consumer, as a large amount of it is lost in both transmission lines and distribution networks. The percentage of these losses ranges from $6 \%$ to $11 \%$, and it reaches much higher in developing countries. The distribution network has the largest share of these losses, as the percentage of losses in it is higher than $70 \%$ of the total losses of the power system. The necessity to reduce the losses of the network due to its negative economic and environmental aspects, as all these losses are additional costs over the network that are paid for by consumers, as well as the losses are directly related to carbon emissions harmful to the environment [1].

One of the ways to reduce losses is Distributed Generation, abbreviated as DG. Distributed generation is a relatively new approach in the field of electric energy. The reference [2] proposes a general definition of it, which is "an electric power source connected directly to the distribution network or on the customer site of the meter".

In addition to reducing losses, there are other advantages of using DG technology such as improving power quality and improving voltage profile, thus, reducing the operation and maintenance costs. Moreover, the use of this technology also postpones the need to install new transmission and distribution lines to meet the growing demand, as well as being characterized by being low in emissions and environmentally friendly.

DG units can also be installed on medium or low voltage distribution networks due to the wide range of sizes that extend from $5 \mathrm{~kW}$ to $300 \mathrm{MW}$ [2]. In addition, it requires a short period of time to install and operate either independently or as a back-up generation, and is not affected by the operating errors of other units. Finally, distributed generation is particularly useful in remote consumers, who 
often suffer from poor network reliability. Due to the previous benefits, electric power systems are changing their electric infrastructure by including many DG technologies. But on the other hand, connecting DG units in some places may cause undesirable behavior on the distribution system, such as: Voltage regulation concerns, Power quality issues, Overloaded situations and Islanding cases [3].

To enhance the DG advantages and to avoid its drawbacks, DG location and its size has to be optimized through optimization problems. These problems are varied according to objectives and solution techniques. Minimizing the total real power losses in the system is one of the most common objectives used in literature. In [4] the study uses a direct approach to obtain load flow solutions and employs particle swarm optimization (PSO) to solve the optimization problem. The proposed technique is investigated by examining the use of diesel generators as DG units in a real distribution system. In order to improve the voltage profile and reduce power losses in a part of a real distribution system, $[5,6]$ uses the Genetic Algorithm (GA). The authors in [7] applied an Ant Colony Optimization (ACO) algorithm for optimal sizing and siting of DGs sizing in a power distribution network. A modified Artificial Bee Colony (ABC) algorithm is proposed in [8] to solve the DG optimization problem, Voltage and thermal constraints are considered and the algorithm was tested on 33-Bus distribution system. A Sequential Quadratic Programming (SQP) deterministic technique was applied in [9] in order to solve a multi-objective DG optimization problem to minimize the both power loss and installation costs of the DG unit. The proposed technique was verified on IEEE 15-bus radial distribution system and the weighed sum model was used to combine the objectives. In [10], the study used Strength Pareto Evolutionary Algorithm (SPEA) and a Nondominated Sorting Genetic Algorithm II (NSGA-II) to solve a multi-objective DG optimization problem to minimize the DG marginal cost and the system's total power losses.

In this paper, a hybrid heuristic technique is proposed to find the best solutions to install a single DG unit in radial distribution systems in order to minimize the total active power losses. The sensitivity test is used to identify the optimal candidate location and the curve fitting test is used to determine the optimal size. The proposed technique is evaluated on IEEE 33-bus and 69-bus and its performance is compared with that of a comprehensive analysis.

\section{Problem Formulation}

The DG optimization problem consists of finding both optimal sizing and suitable placement of the DG unit in the distribution network that would achieve the minimum total active power losses in the system. Thus, the objective function in this paper is minimizing the total real power losses. The thermal power loss of the distribution system can be expressed as a function in line resistances and currents:

$$
\text { Obj.Fun. }=\min : \sum_{i=1}^{n} I_{i}^{2} R_{i}
$$

Where $n$ is the number of branches.

$I i$ is the current magnitude.

$R i$ is the resistance.

$V i$ is the actual voltage at bus $i$.

The minimization of the objective function in equation (1) is subject to several constraints aiming to meet the distributed system requirements and the operational constraints on DG [9]. These constraints can be summarized as follows:

a. The equality constraints are the power flow equations describing the system as follows:

$$
\begin{gathered}
\sum_{i=1}^{n} P_{G i}=\sum_{i=1}^{n} P_{D i}+P_{\text {Loss }} \\
\sum_{i=1}^{n} Q_{G i}=\sum_{i=1}^{n} Q_{D i}+Q_{\text {Loss }}
\end{gathered}
$$

b. The inequality constraints are the system's voltage limits i.e., $\pm 5 \%$ of the nominal voltage value as follows:

$$
\left|V_{i}^{\min }\right| \leq\left|V_{i}\right| \leq\left|V_{i}^{\max }\right|
$$

c. The inequality constraints are the size of the DG which are as follows:

$$
P_{D G i}^{\min } \leq P_{D G i} \leq P_{D G i}^{\max }
$$

\section{Comprehensive Analysis}

The concept of the analysis is based on investigating all possible DG places with all possible sizes, this means that the DG unit will be tested on all buses of the system network one by one, and at each bus, the load flow will be studied several times according to the available sizes of the DG unit, and so on until all possible solutions are investigate. Then, the overall optimal DG option is identified. The methodology is summarized in the following steps:

1. Install the DG unit at the first candidate bus.

2. Choose the minimum size of the DG unit $\left(D G^{\mathrm{min}}\right)$.

3. Run load flow analysis and record the total active power $\left(P_{\text {Loss }}\right)$.

4. Increase the size of the DG unit to the next level and repeat step 3 until reaching the largest size of the DG unit $\left(D G^{\max }\right)$.

5. Install the DG unit to the next bus, and repeat steps 2 to 4 until all candidate buses are tested.

6. The optimal solution (i.e., the optimal placement and sizing of the DG unit) is the one that gives the minimum amount of losses in all tests.

\section{Hybrid Heuristic Technique}

Although the comprehensive analysis studies all possible options for installing the DG unit in the distribution system to choose the best solutions that correspond to the largest reduction in the power losses, the search space for solution is enormous. For example, if a radial distribution system consists of 100 buses and there are 50 different sizes of DG units available, then the required number of tests to solve the optimization problem for this system is 5000. Additionally, if there are two DG units to be installed, the search space will 
double, and become very large. Therefore, some of the common techniques in literature apply simplified assumptions that reduce the search space, such as, fixing the capacity of DG, applying unity power factor DG, and placing the DG unit at the heavily loaded bus in the distribution system [11]. However, in some applications, these methods fail to achieve satisfactory results and may deviate from the goal and not reach the optimal solution. The proposed hybrid heuristic technique consists of two heuristic tests that deal with reducing the size of the search space.

The first stage, in which the best bus is chosen to place the DG unit in the system using sensitivity test, and in the second stage, in which the best size of DG is chosen using curve fitting test.

\subsection{Sensitivity Test}

The sensitivity testing is based on finding the optimal bus for installing DG unit directly without having to check all buses in the distribution system. The idea of the sensitivity test centers on deactivates power demand at each bus once at a time $\left(P_{D i}=0\right)$, and determine the total power losses $\left(P_{\text {Loss }}^{\prime}\right)$ by running load flow analysis, and then, calculate the sensitivity index as follows:

$$
S I_{i}=\frac{P_{\text {LosS }}-P_{\text {LosS }}^{\prime}}{P_{\text {LosS }}}
$$

Where:

$P_{\text {Loss }}$ is the total active power loss for the system.

$P_{\text {Loss }}^{\prime}$ is the total active power when the power demand at bus $(i)$ is deactivated.

The bus with the highest $S I$ value is chosen as the best place to install the DG unit. This test includes the following steps:

1. Run the load flow analysis of the default case, and calculate the total active power loss $P_{\text {Loss }}$.

2. Set the power demand value of the $i$-th candidate bus to zero, $S_{D i=} 0$.

3. Run load flow analysis and record the total active power loss $\left(P_{\text {Loss }}^{\prime}\right)$.

4. Calculate the Sensitivity Index $\left(S I_{i}\right)$ of bus $I$ using equation (6).

5. Repeat steps 2 to 4 with the rest of the candidate buses in the array.

6. The bus in which gives the highest value of the sensitivity index $(S I)$, is chosen as the best place to install the DG unit.

After determining the best location for connecting the DG unit, the best size is determined using the curve fitting test.

\subsection{Curve Fitting Test}

For any distribution network, placement of DG unit will change the real power loss profile of that network. Actually, in a distribution system, the total real power loss is reduced to a minimum value as the DG size is increased. With further increasing of DG size, losses again start to increase [12]. The total active power loss curve with the variation of DG size at any bus is approximately quadratic function. Since the optimal DG size falls within 25 and $75 \%$ of the total load demand, therefore, four selected DG sizes $(10,25,75$ and $90 \%$ of the total load demand) are enough to plot the quadratic function curve. By taking the derivative of this function, the minimum point on this curve can be determined as follow:

$$
\begin{gathered}
f(x)=a x^{2}+b x+c \\
\frac{d}{d x}\left(a x^{2}+b x+c\right)=0 \\
2 a x+b=0 \\
x=-\frac{b}{2 a}
\end{gathered}
$$

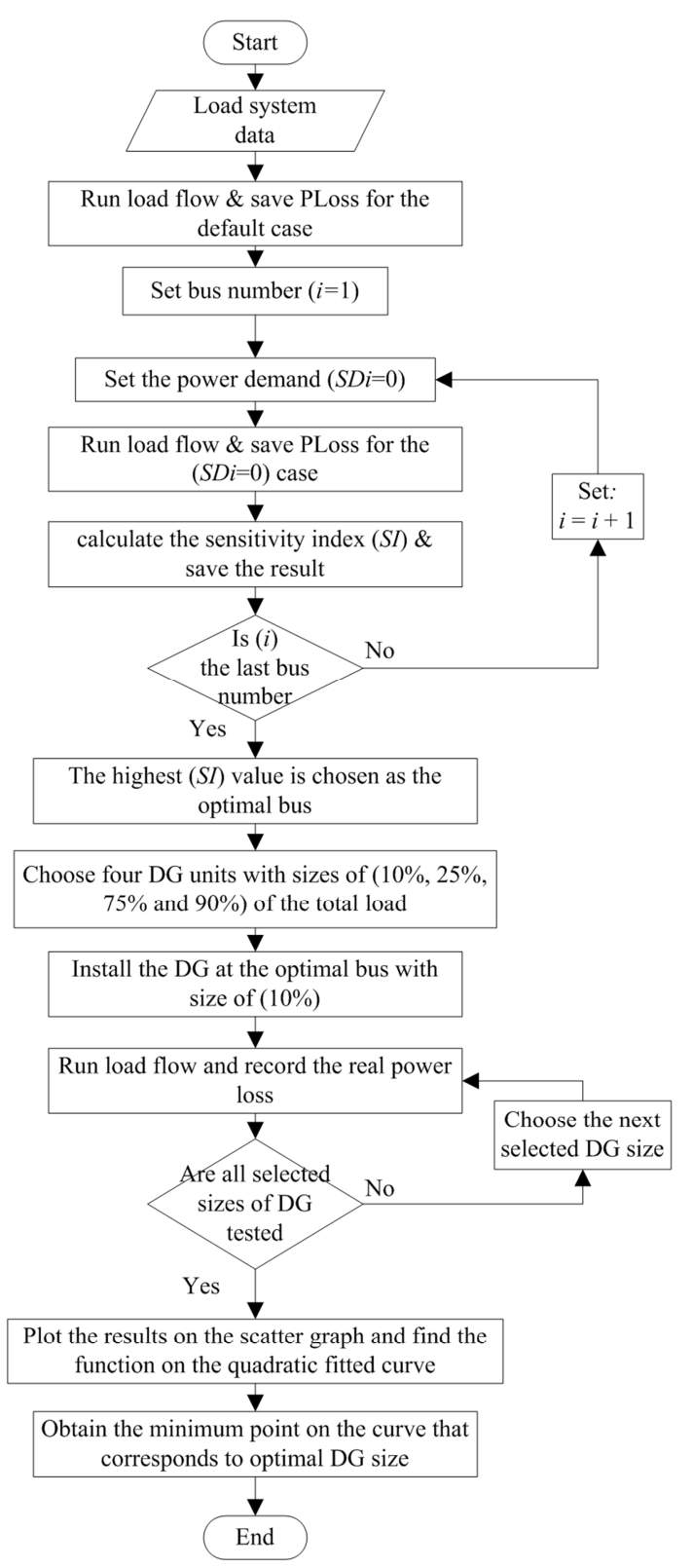

Figure 1. Flowchart of the Proposed Technique.

The curve fitting test is summarized in the following steps:

1. Install the DG unit with the first selected size (10\%) at the first candidate bus.

2. Run load flow analysis and record the total active 
power $\left(P_{\text {Loss }}\right)$.

3. Repeat steps 1 and 2 for the remaining selected sizes of DG $(25 \%, 75 \%, 90 \%)$.

4. Plot the recorded results on a scatter graph where the $x$ axis is the total power loss in the system, and $y$-axis is the DG size.

5. Plot a quadratic fitted curve of the results on the scatter graph and obtain the function of this curve as: $\left(a x^{2}+b\right.$ $x+c)$.

6. Obtain the minimum point at the curve using equation (10), which corresponding to the optimal DG size.

\section{Results and Discussion}

In this section, the presented comprehensive analysis and the hybrid heuristic technique are tested and illustrated using IEEE 33-bus and IEEE-69 bus radial distribution test feeders. The algorithms of both techniques have been developed using MATLAB (R2020b) to ensure the validity of the proposed method in obtaining satisfactory results.

For each distribution system, voltage magnitude and its phase angle for all buses will be found. The voltage profile improvement is evaluated using voltage deviation index which can be obtained by taking the difference between the nominal voltage and the actual voltage. Mathematically, the $\mathrm{VD}$ index is the sum of the squared value of the absolute differences between the nominal and actual voltage for all nodes in the network [13]. This means that lower VD index values indicate better network voltage conditions:

$$
V D=\sum_{i=1}^{i=n}\left|V_{n}-V_{i}\right|^{2}
$$

Where

$n$ is the total number of buses.

$V n$ is the nominal voltage.

$V i$ is the actual voltage at bus $i$.

The DG unit is represented as a negative PQ load that supplies real and reactive power to the distribution network [7]. The DG reactive power can be obtained by the following equation:

$$
Q_{D G}=P_{D G} \times \tan \left(\cos ^{-1}\left(P F_{D G}\right)\right)
$$

Where

$P_{D G}$ is the MW generated by DG unit.

$Q_{D G}$ is the Mvar generated by $\mathrm{DG}$ unit.

$P F_{D G}$ is the power factor of the DG unit.

The power factor of the DG units is assumed to be 0.85 lagging.

\subsection{IEEE 33 Bus Radial Distribution System}

This system, as shown in Figure 2, consists of one main feeder and three laterals, with a total of 33 bus and 32 branches. The main feeder is connected to a $12.66 \mathrm{kV}$ transformer substation. The total registered load of this system is $3715 \mathrm{~kW}$ and 2300 kvar. The total real power loss and the voltage deviation index were determined using power flow calculations, and they are $210.997 \mathrm{~kW}$ and 0.134 respectively.
Data for lines and loads are shown in reference [14].

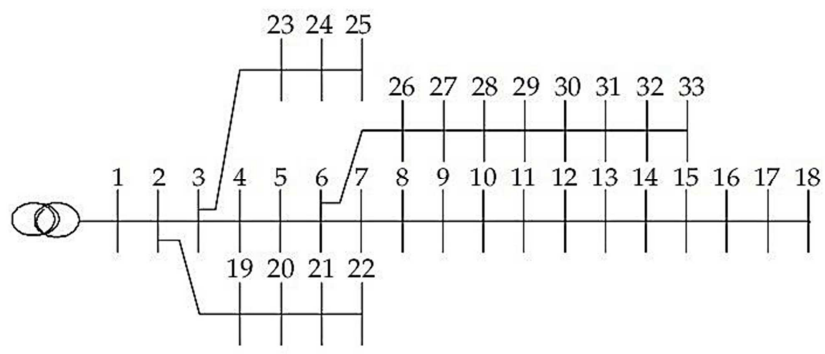

Figure 2. Single Line of IEEE 33-bus Distribution System.

The comprehensive analysis was applied to the distribution system. The number of the DG tests was 832 . Figure 3 shows the search space of the results, the best result was achieved when the DG unit is connecting on bus number 6 with a capacity of $2674.8 \mathrm{~kW}$, which is resulted in the lowest amount of real power losses from $210.997 \mathrm{~kW}$ to $68.1218 \mathrm{~kW}$, i.e. a reduction of $67.70 \%$ and the voltage deviation index is improved from 0.134 to 0.0156 .

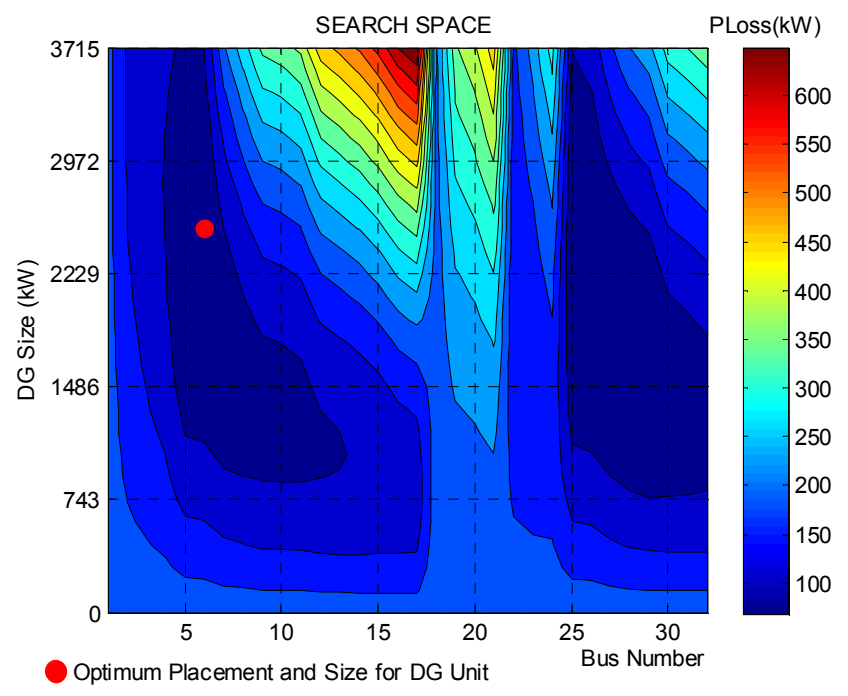

Figure 3. Result of the Comprehensive Analysis for IEEE 33-bus System.

The proposed hybrid technique was also applied to this test system. The results of the sensitivity test, as shown in Figure 4 , indicated that the bus number 30 is the best place to connect the DG unit because it corresponds to the highest sensitivity index value. Accordingly, the curve fitting test was applied to that bus. Based on the selected sizes of the DG units, the quadratic equation was found to be:

$$
P_{\text {Loss }}=199-0.143 P_{D G}+4.07 \times 10^{-5} P_{D G}^{2}
$$

The minimum point on the fitted curve is $1756.25 \mathrm{~kW}$, as shown in Figure 5, which corresponds to the optimal size of the DG unit. The optimal solution reduced the total active power loss of the system from $210.997 \mathrm{~kW}$ to $73.8214 \mathrm{~kW}$ $\mathrm{kW}$, i.e. about $65.00 \%$ reduction. In addition, the voltage deviation index is improved from 0.134 to 0.0293 . 


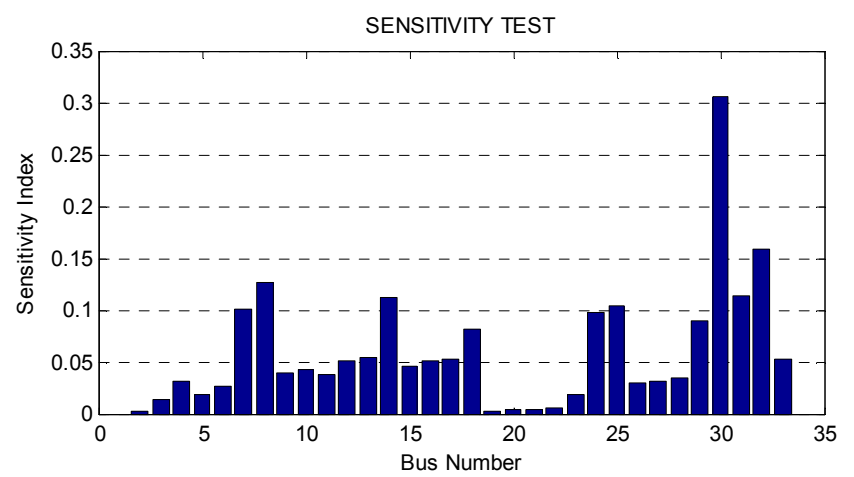

Figure 4. Result of the Sensitivity Test for IEEE 33-bus System.

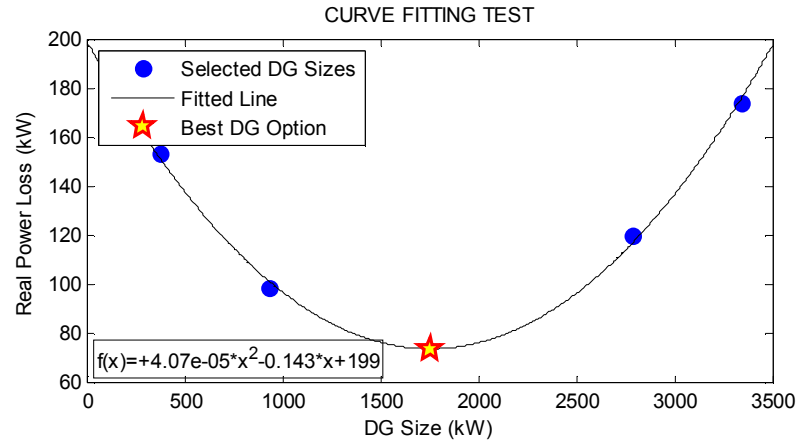

Figure 5. Result of the Curve Fitting Test for IEEE 33-bus System.

Although the result obtained by the comprehensive analysis is better than the result of the hybrid technique, the hybrid technique is characterized by a very small number of tests compared to the number of tests of the comprehensive analysis, as the number of tests in the hybrid technique reached 4 tests, while in the comprehensive analysis it reached 832 tests.
Figures 6 and 7 are visualizing comparison of the results of the voltage profile, real power loss and the voltage deviation index before and after adding the DG unit in the distribution system at the obtained optimal placement and size according to each method. Table 1 lists the optimal solutions achieved by both methods.

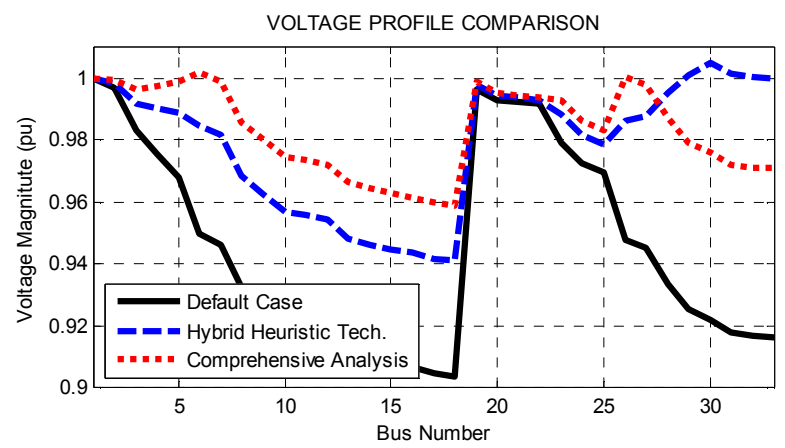

Figure 6. Comparison of Voltage Profile for IEEE 33-bus System.

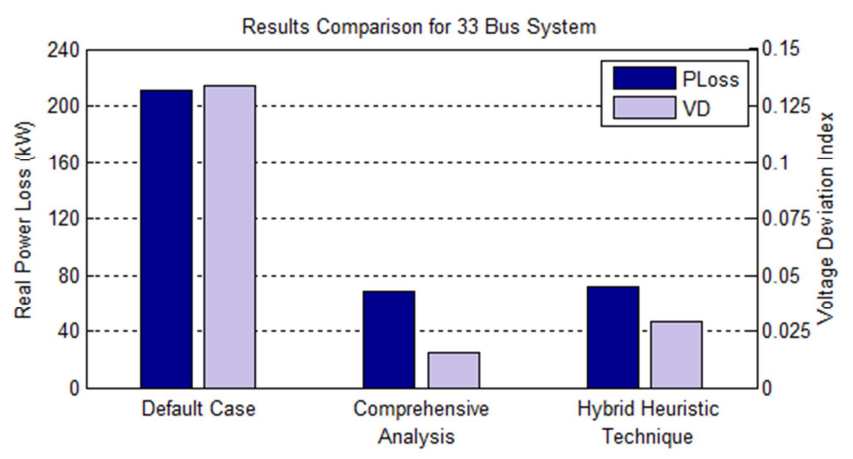

Figure 7. Comparison of Results for IEEE 33-bus System.

Table 1. Results for IEEE 33-bus System.

\begin{tabular}{lll}
\hline Method & Comprehensive Analysis & Hybrid Heuristic Tech. \\
\hline Optimal DG place & bus No. 6 & bus No. 30 \\
Optimal DG size & $2674.8 \mathrm{~kW}$ & $1756.25 \mathrm{~kW}$ \\
$P_{\text {loss }}$ without DG & $210.997 \mathrm{~kW}$ & $210.997 \mathrm{~kW}$ \\
$P_{\text {loss }}$ with DG & $68.1953 \mathrm{~kW}$ & $73.8214 \mathrm{~kW}$ \\
$P_{\text {loss }}$ reduction & $67.70 \%$ & $65 \%$ \\
$V D$ without DG & 0.134 & 0.134 \\
$V D$ with DG & 0.0156 & 0.0293 \\
Total tests & 832 & 4 \\
\hline
\end{tabular}

\subsection{IEEE 69-Bus Radial Distribution System}

The topology of the system is shown in Figure 8. The system consists of one main feeder connected to a transformer substation with voltage of $12.66 \mathrm{kV}$ and seven branched feeders from the main feeder. It contains of 69 buses and 68 branches. The total real and reactive power demand for this system are $3802.19 \mathrm{~kW}$ and $2694.6 \mathrm{kvar}$, respectively. The total active power loss is $225.003 \mathrm{~kW}$, and the voltage deviation index is 0.0994 . The data for lines and loads are listed in reference [15].

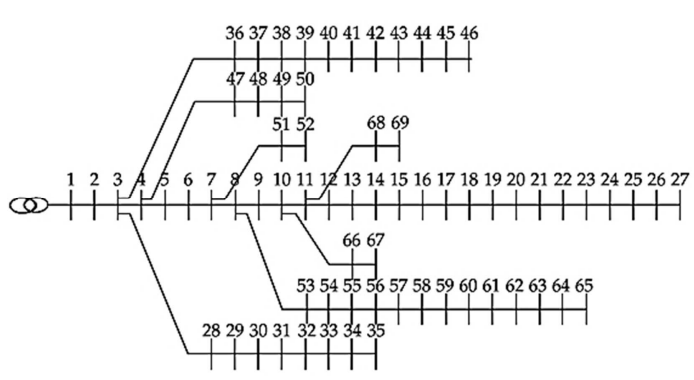

Figure 8. Single Line of IEEE 69-bus Distribution System.

The comprehensive analysis has been applied to the distribution system. From the obtained results as shown in 
Figure 9 and later in Table 2, we note that the search space was increased reaching up to 1768 DG tests. This is a result of the increase in the number of candidate buses for installing DG unit. The best location was at bus number 61 with a DG unit of capacity of $1977.14 \mathrm{~kW}$, this is led to a reduction in the active power loss from $225.003 \mathrm{~kW}$ to $24.1108 \mathrm{~kW}$ by $89.30 \%$ reduction and an improvement in the voltage deviation index from 0.0994 to 0.0112 .

When applying the proposed technique and from the results of the sensitivity test as shown in Figure 10, bus number 61 recorded the highest sensitivity index, therefore, this bus will candidate as the optimal place for installing the DG unit. When applying the curve fitting test to the candidate bus, the quadratic equation was found to be:

$$
P_{\text {Loss }}=210-0.189 P_{D G}+4.76 \times 10^{-5} P_{D G}^{2}
$$

The optimal size of the DG unit is $1984.29 \mathrm{~kW}$, as shown in Figure 11, which reduces the real power losses in the system by $89.30 \%$ from $225.003 \mathrm{~kW}$ to $23.4834 \mathrm{~kW}$. Furthermore, the voltage profile was improved; the voltage deviation index decreased from 0.0994 to 0.0111 .

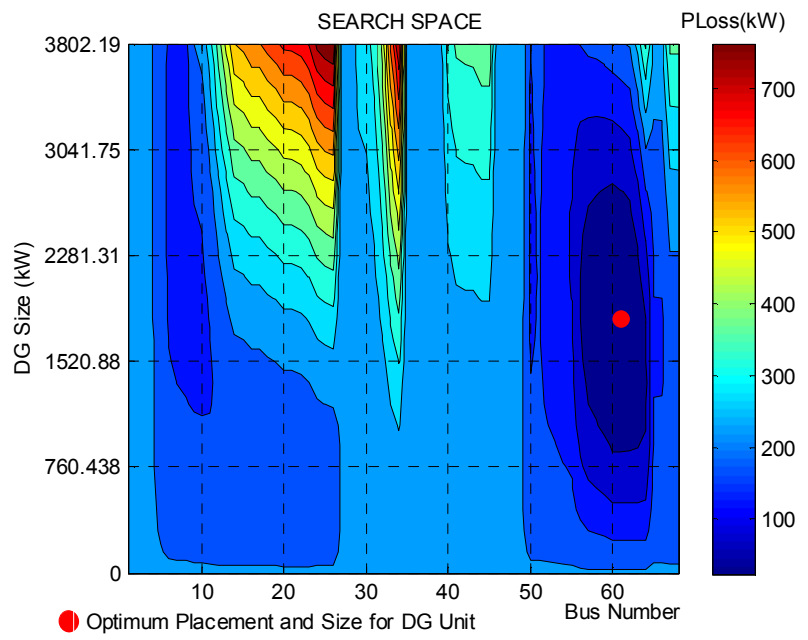

Figure 9. Results of the Comprehensive Analysis for IEEE 69-bus System.

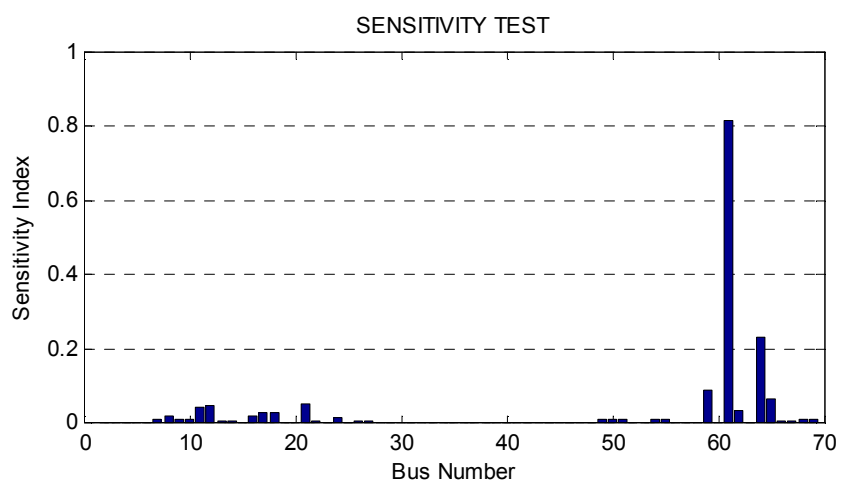

Figure 10. Results of the Sensitivity Test for IEEE 69-bus System.

In the comprehensive analysis, the number of tests increases as the number of buses in the distribution system increases. On the contrary, the number of tests proven and reduced in the hybrid technique regardless of the size of the distribution system. This means that the hybrid technique has effectively reduced the field of research to a very limited number of tests.

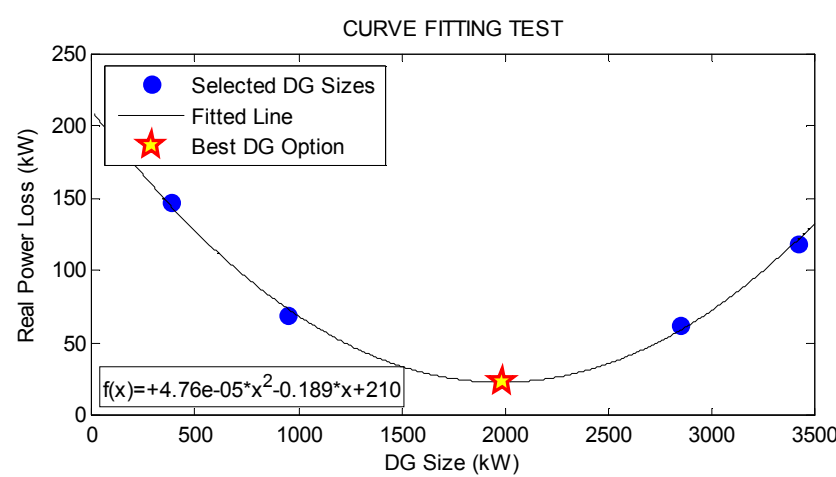

Figure 11. Result of the Curve Fitting Test for IEEE 69-bus System.

Looking at the results obtained from the two methods as shown in table 2 , we note that the reduction in power loss as well as the amount of improvement in the voltage deviation index is almost identical. However, the proposed method outperformed in the small number of the DG tests required to reach the optimal solution. A comparison of the results are shown in Figure 12 and Figure 13, which are include voltage profile, active power loss and voltage deviation index.

Table 2. Results for IEEE 69-bus System.

\begin{tabular}{lll}
\hline Method & Comprehensive Analysis & Hybrid Heuristic Tech. \\
\hline Optimal DG place & bus No. 61 & bus No. 61 \\
Optimal DG size & $1977.14 \mathrm{~kW}$ & $1984.29 \mathrm{~kW}$ \\
$P_{\text {Loss }}$ without DG & $225.003 \mathrm{~kW}$ & $225.003 \mathrm{~kW}$ \\
$P_{\text {Loss }}$ with DG & $24.1108 \mathrm{~kW}$ & $24.1608 \mathrm{~kW}$ \\
$P_{\text {Loss }}$ reduction & $89.30 \%$ & $89.30 \%$ \\
$V D$ without DG & 0.0994 & 0.0994 \\
$V D$ with DG & 0.0112 & 0.0111 \\
Total tests & 1768 & 4 \\
\hline
\end{tabular}

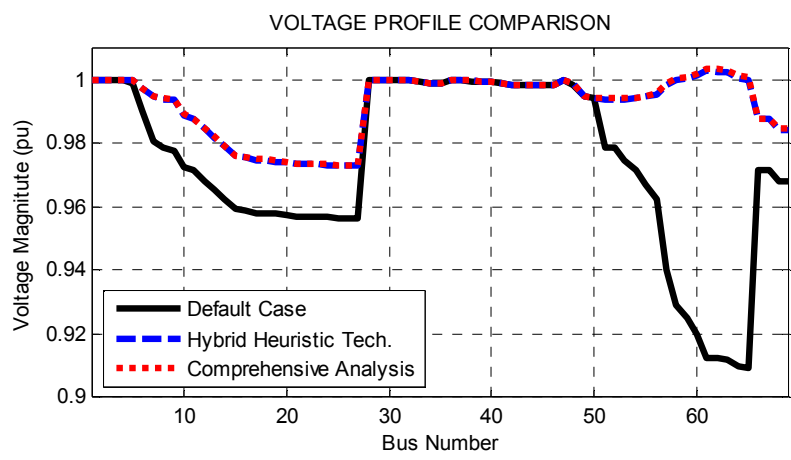

Figure 12. Comparison of Voltage Profile for IEEE 69-bus System.

\section{Conclusion}

In this paper, a hybrid heuristic technique is proposed to reduce real power losses and improve the voltage profile in power systems. The optimal placement of DG is calculated using a sensitivity test, subsequently, the optimal sizing of DG is found using the curve fitting test. A comprehensive analysis has been adopted in order to validate the efficiency 
of the proposed method. Both methods have been tested on IEEE 33-bus and IEEE 69-bus distribution test feeders. The proposed technique successfully identified the optimal solution for the DG optimization problem by reducing the power losses and improving the voltage profile. When tested on IEEE 33-bus system, the comprehensive analysis resulted in $2.7 \%$ more real power loss reduction compared to the hybrid heuristic technique, and a slightly smaller voltage deviation index. On the other hand, the results of both techniques were almost identical when tested on IEEE 69-bus system. However, the proposed technique requires only 4 tests, while the comprehensive analysis requires hundreds of tests depending on the size of the distribution system. Therefore, the hybrid heuristic technique is more efficient as it has a significantly smaller search space. In other words, the proposed technique reduces the computational demand and the convergence time while maintaining satisfactory results.

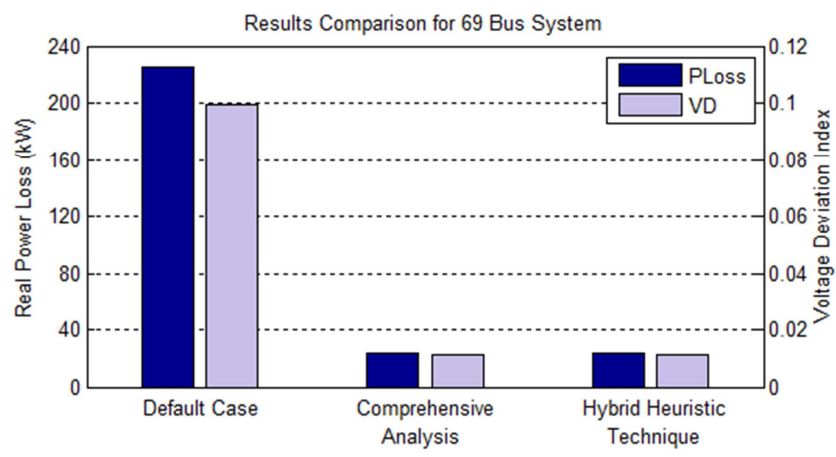

Figure 13. Comparison of Results for IEEE 69-bus System.

Finally, optimal planning for DG units in distribution systems has always been a topic of great interest in power systems research. The proposed hybrid heuristic technique can enhance the performance of radial distribution systems greatly. Therefore, the potential of using this technique in meshed distribution systems should be considered in future research. Additionally, this technique can be further improved by investigating the use of more than one DG unit in larger distribution systems. Moreover, using a multiobjective function instead of a single-objective one is another important method of developing and enhancing the proposed technique. These objectives may give some economic considerations that can reduce the overall costs of the system, or may explore determining the optimal power factor of the DG instead of using a fixed value.

\section{References}

[1] Electricity distribution system losses non-technical overview, Sohn Associates, 2009.

[2] T. Ackermann, G. Andersson and L. Söder, "Distributed generation: A definition," Electr. Power Syst. Res, vol. 57, no. 3, pp. 195-204, 2001.
[3] Abu-Mouti, Fahad Saad. Radial distribution feeders compensation using distributed generation. ProQuest, 2009.

[4] Darfoun, Mohamed. "Improving the Voltage Profile and Minimizing the Power Loss of the Houn $(11 \mathrm{kV})$ Distribution Network." International Conference on Technical Sciences (ICST2019). Vol. 6. 2019.

[5] Sedighizadeh, M., and A. Rezazadeh. "Using genetic algorithm for distributed generation allocation to reduce losses and improve voltage profile." World Academy of Science, Engineering and Technology 37.1 (2008): 251-256.

[6] Kashyap, Mohan, Ankit Mittal, and Satish Kansal. "Optimal placement of distributed generation using genetic algorithm approach." Proceeding of the Second International Conference on Microelectronics, Computing \& Communication Systems (MCCS 2017). Springer, Singapore, 2019.

[7] Ogunsina, Adeseye Amos, et al. "Optimal distributed generation location and sizing for loss minimization and voltage profile optimization using ant colony algorithm." $S N$ Applied Sciences 3.2 (2021): 1-10.

[8] Hussain, Israfil, and Anjan Kumar Roy. "Optimal distributed generation allocation in distribution systems employing modified artificial bee colony algorithm to reduce losses and improve voltage profile." IEEE-International Conference on Advances In Engineering, Science And Management (ICAESM-2012). IEEE, 2012.

[9] Darfoun, Mohamed A., and Mohamed E. El-Hawary. "Multiobjective optimization approach for optimal distributed generation sizing and placement." Electric Power Components and Systems 43.7 (2015): 828-836.

[10] Moeini, A., et al. "Disco planner flexible DG allocation in MV distribution networks using multi-objective optimization procedures." 2010 12th International Conference on Optimization of Electrical and Electronic Equipment. IEEE, 2010.

[11] Abu-Mouti, F. S., and M. E. El-Hawary. "Heuristic curvefitted technique for distributed generation optimisation in radial distribution feeder systems." IET Generation, Transmission \& Distribution 5.2 (2011): 172-180.

[12] Anwar, Adnan, and H. R. Pota. "Loss reduction of power distribution network using optimum size and location of distributed generation." AUPEC 2011. IEEE, 2011.

[13] Mehta, Pankita, Praghnesh Bhatt, and Vivek Pandya. "Optimal selection of distributed generating units and its placement for voltage stability enhancement and energy loss minimization." Ain Shams Engineering Journal 9. 2 (2018): 187-201.

[14] Hajizadeh, Amin, and Ehsan Hajizadeh. "PSO-based planning of distribution systems with distributed generations." International Journal of Electrical and Electronics Engineering 2. 1 (2008): 33-38.

[15] Baran, Mesut E., and Felix F. Wu. "Optimal capacitor placement on radial distribution systems." IEEE Transactions on power Delivery 4. 1 (1989): 725-734. 